Research Article

\title{
Application of an Estimation Method in the Lure System
}

\author{
Zhaoxia Huang (iD \\ Department of Mathematics and Statistics, Ankang University, Ankang 725000, China
}

Correspondence should be addressed to Zhaoxia Huang; 281890896@qq.com

Received 21 September 2020; Revised 22 October 2020; Accepted 16 November 2020; Published 30 November 2020

Academic Editor: Hussein Abulkasim

Copyright (c) 2020 Zhaoxia Huang. This is an open access article distributed under the Creative Commons Attribution License, which permits unrestricted use, distribution, and reproduction in any medium, provided the original work is properly cited.

The presence of set-valued mapping affects the stability of the output of the lure system, adding to the difficulty in observer design. To overcome the difficulty, the author mapped the system output error to the nonlinear term of the framer, creating a framer of the extended Luenberger structure, and analyzed the coordination of the error system by the monotonic system theory. On this basis, the interval observer was designed for the lure system. Then, the lure system and its observer systems were proved as asymptotically stable. Finally, it is proved that the observer system trajectory always followed the original state trajectory through the simulation under the different selections of set-valued mapping.

\section{Introduction}

Due to the wide range of uncertainties in the actual system, the research on uncertainty has received extensive attention. The reasons for the system uncertainty are as follows: modeling error, measurement error, environmental noise, unknown input, and control factors such as failure of the actuator or actuator, external disturbance, and parameter changes. Since the system state cannot be accurately estimated in real time, only the lower and the upper bounds about the state can be given, so the concept of the interval observer is born. The research and application of the interval observer can realize the determination of the state change interval and solve some uncertain problems based on the uncertain method. The main design methods of interval observers so far are limited, and most of them are for linear systems. Since most actual control systems are nonlinear in nature, the interval observer design theory for uncertain nonlinear systems has been developed. It is a very important research significance.

In recent years, there has been more and more research on interval observers, and different research methods have been proposed. The research objects have also expanded from linear time-invariant systems and planar systems to LPV systems, feedback linearization systems, chaotic systems, and Lipschitz nonlinear systems. This chapter mainly studies the design method of the interval observer for the lure nonlinear system. The lure nonlinear system is usually regarded as a relatively simple nonlinear system, but its application is wide and its output is uncertain, which leads to the difficulty of the observer design. This paper will output the error of the system, the nonlinear term of the frame phaser is mapped to the frame phaser of the extended Luenberger structure, and the coordination of the error system is analyzed based on the monotonic system theory. The interval observer design method of the lure system is given, and its algorithm is verified. Effectiveness.

State depicts the information of the dynamic behaviour of a system. To obtain the accurate state of the system is very difficult, due to the presence of various uncertainties. To overcome the difficulty, an effective solution is to take the input and output of the original system as the input to the new system and to use the output of the new system to estimate or reconstruct the state of the original system. The new system is known as the state observer. Many state observers were designed to eliminate the random disturbances [1-5], including several robust designs under bounded uncertainty [6-12].

With set-valued mapping, the lure system has uncertain output, which is a new type of uncertainty. In this paper, an interval observer method was proposed based on the lure system, aiming to disclose the relationship between system 
structure and framer $(\underline{x}(t) / \bar{x}(t))$ configuration and find the way to worsen the state and minimize $x^{+}(t)-x^{-}(t)$. Specifically, the system output error was mapped to the nonlinear term of the framer, producing a framer of the extended Luenberger structure. Meanwhile, the coordination of the error system was analyzed by the monotonic system theory. On this basis, the interval observer design of the lure system was put forward and verified by an algorithm.

\section{Problem Description}

Suppose there is a system with lure type

$$
\begin{aligned}
& \dot{x}(t)=A x(t)+B r(t)-G \omega(t), \\
& \omega(t) \in v(H x(t)), \\
& y(t)=C x(t),
\end{aligned}
$$

where, $x(t) \in R^{n}$ is the state, $y(t) \in R^{r}$ is the output, and $r(t) \in R^{m}$ is the input of the system; A, B, and C are real number matrices with proper dimensions; $G \in R^{n \times q}$ is the output matrix with proper dimensions; $v(\cdot)$ is the set-valued mapping; $H \in R^{q \times n}$ is the input matrix; and $\omega(t) \in R^{q}$ is the output of the set-valued mapping. Here, we assume $B$ and $G$ are column full rank, and $C$ is row full rank. The set-valued mapping is defined below with an example.

As the fundamental concept of the differential inclusion theory, set-valued mapping was further described as follows.

Set-valued mapping exists in many actual systems. A typical example is the friction in mechanical systems and the diode in electronic systems. Set-valued mapping is needed to describe these basic elements in a specific manner. Hence, set-valued mapping and the ensuing differential inclusion are indispensable for the creation of an accurate model for dynamic systems.

In 1902, Stribeck discovered the variation curve of the friction coefficient when he explored dry friction (i.e., the friction without lubricant). This curve is now known as the Stribeck curve Figure 1. With the change in speed, the variation in friction coefficient goes through four phases, namely, static friction, Stribeck friction, Coulomb friction, and viscous friction. In the phase of static friction, there is no relative motion between the friction pairs; in the phase of Stribeck friction, relative motion occurs between the friction pairs, and the friction coefficient gradually declines. The four-phase process exhibits an obvious nonlinearity. Setvalued mapping appears in the phase of static friction. Despite the absence of relative motion, the friction force still exists in the same magnitude as the external force but points to the opposite direction.

Glocker described the friction force $\lambda$ with the following formula of set-valued mapping:

$$
\lambda \in F_{f}(\dot{q})=-\mu \lambda_{N} \operatorname{sgn}(\dot{q})+F_{S}(\dot{q}),
$$

where $F_{S}(\dot{q})$ is a function of the speed; $q$ is the displacement; $\mu$ is the friction coefficient; and $\lambda_{N}$ is the positive pressure; we view $\operatorname{sgn}(\dot{q})$ as a set-valued mapping:

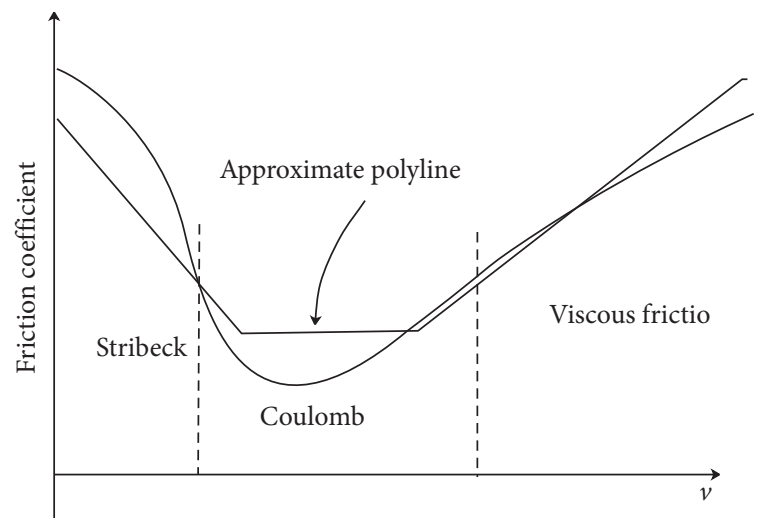

FIgURE 1: Stribeck curve.

$$
\operatorname{sgn}(\dot{q})= \begin{cases}1, & \dot{q}>0 \\ {[-1,1],} & \dot{q}=0 \\ -1, & \dot{q}<0 .\end{cases}
$$

Only a controlled mechanical system with dry friction can be described as follows:

$$
M \ddot{q}+D \dot{q}+K q=S u+T \lambda,
$$

where $K, D$, and $M$, are the stiffness matrix, damping matrix, and mass matrix of the mechanical system; $q$ is the generalized speed containing both translation and rotation; $S$ is an input matrix; and we assume $u$ as the control variable; $T$ is the friction matrix; $\lambda$ is the vector composed of friction forces. The $i$ th component of $\lambda$ stands for the friction at the $i$ th contact point, which can be expressed as the set-valued mapping below:

$$
\lambda_{i} \in-\mu_{i} \lambda_{N i} \operatorname{sgn}\left(T_{i}^{T} \dot{q}\right)+F_{S i}\left(T_{i}^{T} \dot{q}\right)
$$

where $T_{i}$ is the $i$ th column of $T$ and $T_{i}^{T} \dot{q}$ is the relative sliding of the $i$ th contact point. The $M \ddot{q}+D \dot{q}+K q=S u+T \lambda$ can be expressed as the following state space:

$$
\begin{aligned}
& \dot{x}=A x+G w+B u, \\
& z=H x, \\
& y=C x, \\
& w \in-\varphi(z),
\end{aligned}
$$

where $x=\left(q^{T} \dot{q}^{T}\right)^{T}$ is the state; $w$ and $z$ are the output and input of $\varphi(\cdot)$; and $y$ is the measurable output. It can be seen that set-valued mapping is another form of uncertainty. This paper focuses on the design of interval observers for the systems with such uncertainty.

The differences between differential inclusion and traditional differential equations are described below.

Let $f:[0, T] \times R^{n} \longrightarrow R^{n}$ be a known single-valued mapping $(T \in R(>0)), t \in[0, \mathrm{~T}]$ be time, $x(t)$ be an unknown single-valued mapping of $[0, T] \longrightarrow R^{n}$, and $\dot{x}(t)$ be a derivative of $x(t)$ relative to $t$.

If $F:[0, T] \times R^{n} \longrightarrow R^{n}$ is a set-valued mapping, $x(t)$ is an unknown function of $[0, T] \longrightarrow R^{n}$. The solving of $x(t)$ from $\dot{x}(t) \in F(t, x(t))$ is a differential inclusion problem. If 
$F$ is only $R^{n} \longrightarrow R^{n}$, without explicitly containing $t$, then the differential inclusion becomes $\dot{x}(t) \in F(x(t))$.

The above formula is known as the time-invariant differential inclusion, while the previous formula is known as the time-varying differential inclusion.

If there is a Luenberger observer satisfying (1), then $\mathrm{H}$ must be row full rank. In the lure system, we assume the setvalued mapping $v(\cdot)$ as monotonic. In other words, $\omega_{1}-\omega_{2}, H x_{1}-H x_{2} \geq 0$.

The author further investigated the Cauchy problem of (1) and (2) under the initial condition of $x(0)=x_{0}$ with $\omega \in v(\cdot)$. For the design of differential inclusion observer, the adaptive problem can be solved by two methods. First, it is assumed that, for any $\omega(t) \in v(H x(t))$, the equation $\dot{x}(t)=A x(t)+B r(t)-G \omega(t), \quad x(0)=x_{0}$, or that the setvalued mapping $v(\cdot)$ is a closed convex function with Lipshitz continuity. Second, we assume $v(\cdot)$ as maximal monotonic, such that the solution not only exists for $\dot{x}(t)=A x(t)+B r(t)-G \omega(t), x(0)=x_{0}$, but also is unique except for a zero measure set.

The lure type differential inclusion system has a closedloop structure. The nonlinear part is concentrated on the feedback channel. It can be viewed as the product of nonlinear feedback of a linear system, provided that the nonlinear feedback satisfies the Popov integral inequality, i.e., $\int_{t_{0}}^{t} v^{T}(\tau) y(\tau) d_{\tau} \geq-r_{0}^{2}$ with $v$ being the output of the nonlinear part and $y$ being the output of the linear part.

\section{Nature of Set-Valued Mapping}

In the first two selections, the single-valued mapping is either continuous or measurable. In the approximate selection, a function sequence with good properties is selected such that it approximates $\operatorname{clF}(x)$.

Lemma 1. If set-valued mapping $v(\cdot): R^{m} \longrightarrow R^{m}$ is maximal monotonic, then $R^{n} \longrightarrow R^{n}$ 's mapping $x \mapsto H^{T} v(H x+h)$ is monotonic, $x \in R^{n}, h \in R^{m}, H \in R^{m \times n}$, and $H$ is row full rank.

Proof. Let $y_{i} \in x \mapsto H^{T} v(H x+h) i=1,2$. Then, there exists $\omega_{i} \in v\left(H x_{i}+h\right)$ such that $y_{i}=H^{T} \omega_{i}$. Since $H$ is row full rank, we only have to consider $\omega_{i}$ after determining $y_{i}$. Considering that

$$
\begin{aligned}
& \left\langle y_{1}-y_{2}, x_{1}-x_{2}\right\rangle=\left\langle H^{T} \omega_{1}-H^{T} \omega_{2}, x_{1}-x_{2}\right\rangle=\left\langle\omega_{1}-\omega_{2}, H x_{1}-H x_{2}\right\rangle \\
& \left\langle\omega_{1}-\omega_{2},\left(H x_{1}+h\right)-\left(H x_{2}+h\right)\right\rangle=\left\langle\omega_{1}-\omega_{2}, H x_{1}-H x_{2}\right\rangle \geq 0
\end{aligned}
$$

we have $y_{1}-y_{2}, x_{1}-x_{2} \geq 0$; that is, $x \mapsto H^{T} v(H x+h)$ is monotonic.

The next step is to prove the maximal monotonicity of $x \mapsto H^{T} v(H x+h) \cdot v(H x+h)$ is maximal monotonic. If $v(H x)$ is monotonic, we only need to prove that $x \mapsto H^{T} v(H x)$ is maximal monotonic.

Let $y_{1} \in H^{T} v\left(H x_{1}\right)$. If $y_{2} \in \operatorname{lm} H^{T}$, there exists $x_{2} \in R n$ such that $y_{1}-y_{2}, x_{1}-x_{2} \geq 0$. Thus $y_{2} \in \operatorname{lm} H^{T}$, and there exists $\omega_{2} \in R m$ such that $y_{2}=H^{T} \omega_{2}$. Since $y_{1} \in H^{T} v\left(H x_{1}\right)$, there exists $\omega_{1} \in v\left(H x_{1}\right)$ such that $y_{1}=H^{T} \omega_{1}$. Hence, $y_{1}-$ $y_{2}, x_{1}-x_{2} \geq 0$ is $\omega_{1}-\omega_{2}, H x_{1}-H x_{2} \geq 0$. Since $v(H x)$ is maximal monotonic, we have $\omega_{2} \in v\left(H x_{2}\right)$; that is, $y_{2} \in H^{T} v\left(H x_{2}\right)$.

Lemma 2. The linear and nonlinear parts of the loop in the lure type differential inclusion system are separable. If the nonlinear part satisfies the Popov integral inequality, then the necessary and sufficient condition for the closed-loop system to be ultrastable is that the linear part is positive and real; that is, the transfer function matrix is positive and real.
Proof. Adequacy. Let $V(x)=x^{T} P x$ be a positive definite function. We have

$$
\begin{aligned}
\dot{V}(x)= & (A x-B v)^{T} P x+x^{T} P(A x-B v) \\
= & x^{T}\left(A^{T} P+P A\right) x-v^{T} B^{T} P x-x^{T} P B v \\
= & -x^{T} L^{T} L x-2 v^{T}\left(C-K^{T} L^{T}\right) x \\
= & -x^{T} L^{T} L x+2 v^{T} K^{T} L^{T}-v^{T} K^{T} K v-2 v^{T} C x \\
& +v^{T} K^{T} K v \\
= & -\|L x-K v\|^{2}-v^{T} C x-x^{T} C^{T} v+v^{T} D v+v^{T} D^{T} v \\
= & -\|L x-K v\|^{2}-2 v^{T} y \leq-2 v^{T} y .
\end{aligned}
$$

Through the integration on both sides, we have

$$
\int_{0}^{t} \dot{V} d_{t}=x^{T}(t) P x(t)-x^{T}(0) P x(0) \leq-2 \int_{0}^{t} v^{T}(\tau) y(\tau) d_{t} \leq 2 r_{0}^{2}
$$

According to the inequality above, we have 


$$
\lambda_{m}\|x(t)\|^{2} \leq \lambda_{M}\|x(0)\|^{2}+2 r_{0}^{2} \leq K\left(\|x(0)\|^{2}+r_{0}^{2}\right) \leq K\left(\left\|x(0)+r_{0}\right\|\right)^{2}
$$

where $K=\max \left(\lambda_{M}, 2\right)$. Thus, we have $x\left(t, x_{0}\right.$, $\left.t_{0}\right) \leq K\left(x_{0}+r_{0}\right)$, indicating that the system is ultrastable.

Necessity. If there exists $\sigma_{0}+i \omega_{0}$, in which $\sigma_{0}>0$, such that $G\left(\sigma_{0}+i \omega_{0}\right)+G^{*}\left(\sigma_{0}+i \omega_{0}\right)$ is not positive semidefinite. In other words, there exists $u_{0} \in C m$, where $\mathrm{C}$ is a complex number field, $u_{0}=1$, and $u_{0}^{*}\left[G\left(\sigma_{0}+i \omega_{0}\right)+G^{*}\right.$ $\left.\left(\sigma_{0}+i \omega_{0}\right)\right] u_{0}<0$. Under the input $u(t)=\operatorname{Re} u_{0} e^{\left(\sigma_{0}+i \omega_{0}\right) t}$, since $\mathrm{G}(\mathrm{s})$ means the system is asymptotically stable and $f(t)=\sum_{k} \operatorname{Re} s F\left(p_{k}\right) e^{p_{k} t} y(t)=\operatorname{Re} G\left(\sigma_{0}+i \omega_{0}\right) u_{0} e^{\left(\sigma_{0}+i \omega_{0}\right) t}$

Considering $u^{T}(t) y(t)=\operatorname{Re} u_{0}^{T} e^{\left(\sigma_{0}+i \omega_{0}\right) t} \operatorname{Re} G\left(\sigma_{0}+i \omega_{0}\right)$ $u_{0} e^{\left(\sigma_{0}+i \omega_{0}\right) t}$, it can be concluded that $\int_{0}^{t} u^{T}(\tau) y(\tau) d_{\tau} \longrightarrow \infty$ through in-depth discussion. Thus, the Popov integral inequality $\int_{t_{0}}^{t} v^{T}(\tau) y(\tau) d_{\tau} \geq-r_{0}^{2}$ is valid.

\section{Design Method for Lure System State Observer}

For the lure type differential inclusion system (1), the Luenberger interval observers can be designed as follows:

$$
\begin{aligned}
& \dot{x}^{+}=A x^{+}+B r(t)+L\left(y-C x^{+}\right)-G \omega^{+}(t), \\
& \dot{x}^{-}=A x^{-}+B r(t)+L\left(y-C x^{-}\right)-G \omega^{-}(t),
\end{aligned}
$$

where $\omega(t) \in v(H x(t))$, with $x$ being the state and $\omega$ being the output of set-valued mapping; $L \in R^{n \times r}$ is the observation gain which ensures that $A-L C$ is a Hurwitz matrix. According to the linear system, when $(A, C)$ can be observed, there $A-L C$ is a Hurwitz matrix.

Subtracting $\dot{x}(t)=A x(t)+B r(t)-G \omega(t)$ from $\dot{x}^{+}=$ $A x^{+}+B r(t)+L\left(y-C x^{+}\right)-G \omega^{+}(t)$, we have

$$
\begin{aligned}
\dot{x}^{+}-\dot{x} & =(A-L C) x^{+}+G \omega(t)-G \omega^{+}(t), \\
\omega(t) & \in v(H x(t)), \\
\omega^{+}(t) & \in v\left(H x^{+}(t)\right), \\
y(t) & =C x(t) .
\end{aligned}
$$

Let $e^{+}=x^{+}-x$ be the observer error. Then, the above formula can be rewritten as follows:

$$
\begin{aligned}
\dot{e}^{+} & =(A-L C) x^{+}+G\left(\omega(t)-\omega^{+}(t)\right), \\
\omega(t) & \in v(H x(t)), \\
\omega^{+}(t) & \in v\left(H x^{+}(t)\right) .
\end{aligned}
$$

Similarly, subtracting $\dot{x}^{-}=A x^{-}+B r(t)+L\left(y-C x^{-}\right)$ $-G \omega^{-}(t)$ from $\dot{x}(t)=A x(t)+B r(t)-G \omega(t)$, we have

$$
\begin{aligned}
\dot{x}-\dot{x}^{-} & =(A-L C) x^{-}+G \omega^{-}(t)-G \omega(t), \\
\omega(t) & \in v(H x(t)), \\
\omega^{-}(t) & \in v\left(H x^{-}(t)\right), \\
y(t) & =C x(t) .
\end{aligned}
$$

Let $e^{-}=x-x^{-}$be the observer error. Then, the above formula can be rewritten as follows:

$$
\begin{aligned}
\dot{e}^{-} & =(A-L C) x^{-}+G\left(\omega^{-}(t)-\omega(t)\right), \\
\omega(t) & \in v(H x(t)), \\
\omega^{-}(t) & \in v\left(H x^{-}(t)\right) .
\end{aligned}
$$

To find the $L$ that makes the error system stable is our aim.

Lemma 3. For differential inclusion systems (1)-(3), (13), and (14) are the Luenberger interval observers of the system (1):

(i) There exists an $L$ such that $(H, A-L C, G)$ is observable, strictly positive and real and controllable

(ii) $v(\cdot)$ is monotonic

(iii) The differential inclusions (16) and (18) have solutions

Proof. Let $z=H x, \widehat{z}^{+}=H \hat{x}^{+}$, and $\widehat{z}^{-}=H \widehat{x}^{-}$be the auxiliary outputs of the system (1), interval observer (13), and interval observer (14), respectively. Since $v(\cdot)$ is monotonic, we have $\omega-\omega^{+}, z-z^{+} \geq 0$ and $\omega^{-}-\omega, z^{-}-z \geq 0$. Let $\mu_{1}=\omega-\omega^{+}$, $\mu_{2}=\omega^{-}-\omega, \zeta_{1}=H\left(x^{+}-x\right)$, and $\zeta_{2}=H\left(x-x^{-}\right)$. Then, (16) and (18) can be expressed as follows:

$$
\begin{aligned}
& \dot{e}^{+}=(A-L C) x^{+}+G \mu_{1}, \\
& \zeta_{1}=H e^{+}, \\
& \mu_{1} \in v(H x)-v\left(H x^{+}\right), \\
& \dot{e}^{-}=(A-L C) x^{-}+G \mu_{2}, \\
& \zeta_{2}=H e^{-} \\
& \mu_{2} \in v\left(H x^{-}\right)-v(H x) .
\end{aligned}
$$

Let

$v\left(e^{+}, x\right)=v\left(H x^{+}\right)-v(H x)=v(H x)-v\left(H\left(x-e^{+}\right)\right)$.

Obviously, $0 \in v(0, x)$ is valid for all $x$. Let $\mu_{1}$ and $\mu_{2}$ be the inputs of the linear parts of systems (16) and (18), respectively, and $\xi_{1}$ and $\xi_{2}$ be the outputs of the linear parts of systems (16) and (18), respectively. Then, $\omega-\omega^{+}, z-z^{+} \geq 0$, and $\omega^{-}-\omega, z^{-}-z \geq 0$ imply that $\mu_{1}, \zeta_{1} \geq 0$ and $\mu_{2}, \zeta_{2} \geq 0$. According to Lemma 2, $(H, A-L C, G)$ is real and strictly positive. Meanwhile, $e^{-} \longrightarrow 0$ and $e^{+} \longrightarrow 0$, indicating that all dynamic systems are asymptotically stable and positive. Thus, we have $x^{-} \leq x \leq x^{+}$. This proves that (13) and (14) are interval observers of the system (1).

Lemma 4. Let $P$ be a given $m \times n$ real symmetric matrix, $U \in R^{m \times n}$, and $V \in R^{m \times i}$ be given column full rank matrices, and $U_{\perp} V_{\perp}$ be the orthogonal complement matrix of $U$ and $V$, respectively. Then, the necessary and sufficient conditions for the existence of a $Q$, such that

$$
P+U Q V^{T}+V Q^{T} U^{T}<0
$$


are

$$
\begin{aligned}
& \left(V_{\perp}\right)^{T} P V_{\perp}<0, \\
& \left(U_{\perp}\right)^{T} P U_{\perp}<0 .
\end{aligned}
$$

The significance of Lemma 3 lies in $V$ and $U$ that appear in the same term in $P+U Q V^{T}+V Q^{T} U^{T}<0$, when matrix $Q$ is unknown. By contrast, $U_{\perp}$ and $V_{\perp}$ appear in two independent inequalities, when matrix $Q$ is known. This makes the problem easier to solve. The proof of the lemma and the general formula of $Q$ satisfying $P+U Q V^{T}+V Q^{T} U^{T}<0$ are available in [13-17].

$U$ is a reversible matrix because it is column full rank. In this case, $U_{\perp}$ is an element zero, and $\left(U_{\perp}\right)^{T} P U_{\perp}<0$ should be removed from the lemma.

If $U$ is a reversible matrix, the unique $Q$ can be determined by $K=U Q$. In this case, if $K$ is set to $k V$, then

$$
P+U Q V^{T}+V Q^{T} U^{T}=P+2 k V V^{T} .
$$

According to the Finsker theorem, the necessary and sufficient condition for the existence of an $k$ such that $P+$ $2 k V V^{T}<0$ is $V_{\perp}^{T} P V_{\perp}<0$ [18].

Thus, we have the following theorem:

Theorem 1. If $G$ is column full rank, the necessary and sufficient conditions for the existence of an $L$ such that $(H, A-L C, G)$ is strictly positive and real and are as follows:

(1) $H G>0$, i.e., $H$ is row full rank

(2) $M_{\perp}^{T} C_{\perp}\left(N A+A^{T} N\right) C_{\perp}^{T} M_{\perp}<0$ and $\left(C_{\perp} A^{T} G_{\perp}\right) C_{\perp}^{T} C_{\perp}\left(N A+A^{T} N\right) C_{\perp}^{T} C_{\perp} A^{T} G_{\perp}<0$, where $M=C_{\perp} G_{\perp}$ and $N=H^{T}(H G)^{-1} H$

Conversely, if the lemma conditions are valid, we can obtain a $P$ such that $G^{T} P=H$. According to condition (13) of the lemma, we have $C_{\perp}\left(P A+A^{T} P\right) C_{\perp}^{T}<0$. Then, it can be seen from Lemma 3 that $(H, A-L C, G)$ is strictly positive and real.
Note that the unknown term in the inequality of condition (13) is the matrix $X$, and the inequality is linear relative to $X$. Thus, the inequality tool of the linear matrix can be adopted for the solution.

For practice, a simpler method is mentioned to solve the inequality $C_{\perp}\left(P A+A^{T} P\right) C_{\perp}^{T}<0$ independently, which is irrelevant to $G^{T} P=H$.

Further results can be derived from Lemma 3 through the analysis of

$$
\begin{aligned}
C_{\perp} & {\left[\left(H^{T}(H G)^{-1} H+G_{\perp} X G_{\perp}^{T}\right) A\right.} \\
& \left.+A^{T}\left(H^{T}(H G)^{-1} H+G_{\perp} X G_{\perp}^{T}\right)\right] C_{\perp}^{T}<0 .
\end{aligned}
$$

For simplicity, let $M=C_{\perp} G_{\perp}$ and $N=H^{T}(H G)^{-1} H$. Then, we have $M \in R^{(n-r) \times(n-q)}, N \in R^{n \times n}$, and $N$ is a semipositive definite. Then, the above inequality can be rewritten as follows:

$$
C_{\perp}\left(N A+A^{T} N\right) C_{\perp}^{T}+M X G_{\perp}^{T} A C_{\perp}^{T}+C_{\perp} A^{T} G_{\perp} X M^{T}<0 .
$$

According to Lemma 3, the necessary and sufficient conditions $C_{\perp}\left(N A+A^{T} N\right) C_{\perp}^{T}+M X G_{\perp}^{T} A C_{\perp}^{T} C_{\perp}^{T}+C_{\perp} A^{T} G_{\perp} X M^{T}<0$ to be valid are

$$
\begin{array}{r}
M_{\perp}^{T} C_{\perp}\left(N A+A^{T} N\right) C_{\perp}^{T} M_{\perp}<0, \\
\left(C_{\perp} A^{T} G_{\perp}\right) C_{\perp}^{T} C_{\perp}\left(N A+A^{T} N\right) C_{\perp}^{T} C_{\perp} A^{T} G_{\perp}<0 .
\end{array}
$$

\section{Simulation}

The following lure system is established for simulation:

$$
\left[\begin{array}{l}
\dot{x}_{1} \\
\dot{x}_{2} \\
\dot{x}_{3}
\end{array}\right]=\left[\begin{array}{ccc}
-10 & -3 & -1 \\
6 & -5 & 4 \\
1 & 0 & -9
\end{array}\right]\left[\begin{array}{l}
x_{1} \\
x_{2} \\
x_{3}
\end{array}\right]-\left[\begin{array}{l}
1 \\
2 \\
2
\end{array}\right] \omega+\left[\begin{array}{l}
2 \\
0 \\
1
\end{array}\right] r,
$$

where $y=x_{1}, v(\cdot)$ can be expressed as follows:

$$
\begin{aligned}
& v\left(x_{1}+3 x_{2}+2 x_{3}\right)= \begin{cases}x_{1}+3 x_{2}+2 x_{3}+3 \operatorname{sgn}\left(x_{1}+3 x_{2}+2 x_{3}\right), & x_{1}+3 x_{2}+2 x_{3} \neq 0, \\
{[-3,3],} & x_{1}+3 x_{2}+2 x_{3}=0,\end{cases} \\
& A=\left[\begin{array}{ccc}
-10 & -3 & -1 \\
6 & -5 & 4 \\
1 & 0 & -9
\end{array}\right], \\
& B=\left[\begin{array}{l}
2 \\
0 \\
1
\end{array}\right] \text {, } \\
& C=\left[\begin{array}{lll}
1 & 0 & 0
\end{array}\right] \text {, } \\
& G=\left[\begin{array}{l}
1 \\
2 \\
2
\end{array}\right] \text {. }
\end{aligned}
$$

$$
\text { Let } L=\left[\begin{array}{lll}
-6 & 2 & 1
\end{array}\right]^{T} \text {. We have } A-L C=\left[\begin{array}{ccc}
-4 & -3 & -1 \\
4 & -5 & 4 \\
0 & 0 & -9
\end{array}\right] \text {. }
$$




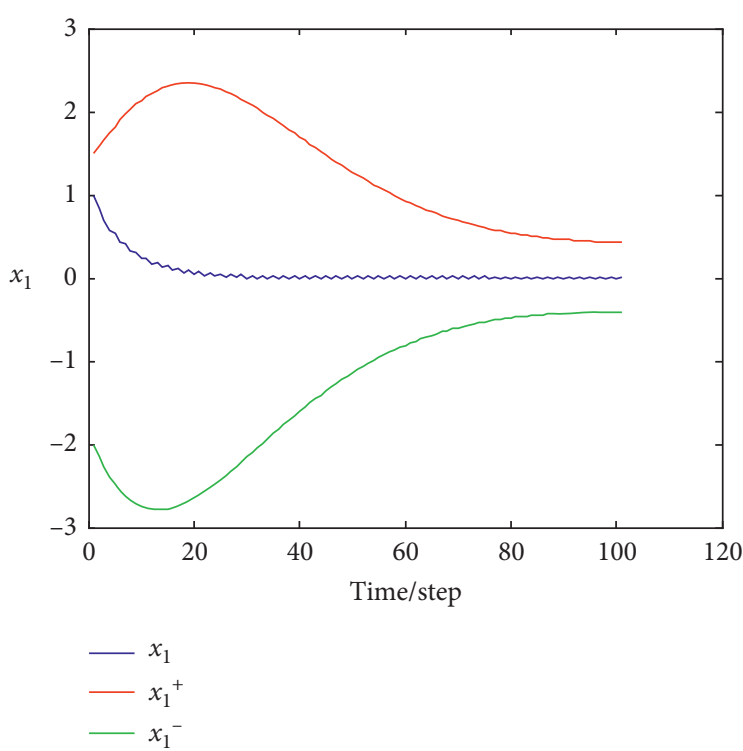

(a)

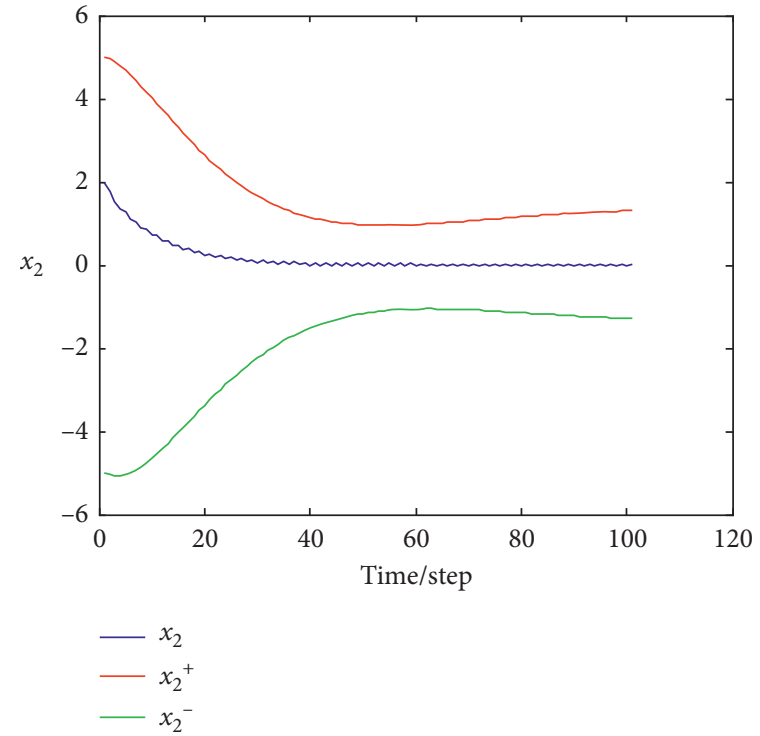

(b)

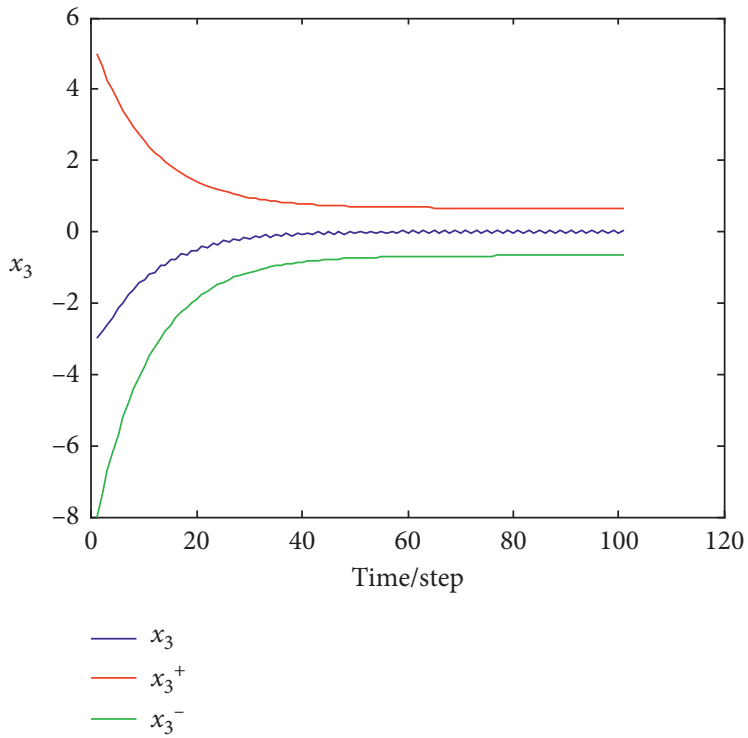

(c)

FiguRE 2: State trajectories of the lure system and the interval observers. It can be calculated that $P=\left[\begin{array}{ccc}1 & 0 & 0 \\ 0 & 1 & 0.5 \\ 0 & 0.5 & 0.5\end{array}\right]$ and
$H=\left[\begin{array}{lll}1 & 3 & 2\end{array}\right]$.

Figure 2 shows the estimated interval state of the system. It can be seen that $x_{i}^{-}(t) \leq x_{i}(t) \leq x_{i}^{+}(t), i=1,2,3$ at any time. This means the proposed interval observers (13) and (14) can estimate the interval state of the system well.

As shown in Figure 2, the lure system and its observer systems were asymptotically stable under different selections of set-valued mapping, and the observer system trajectory always followed the original state trajectory.

\section{Conclusions}

This paper explores the interval observer design in the lure system. Interval state estimation is a hot and frontier content in control theory research, and it has a wide range of applications in many engineering fields. At present, the international research on interval observers has just started, and the research objects are mainly focused on linear systems, with limited research methods and conclusions. Therefore, to carry out research on interval observers and control of uncertain systems not only has important 
scientific significance and theoretical innovation but also has a certain engineering application value.

The presence of set-valued mapping affects the stability of the output of the lure system, adding to the difficulty in observer design. To overcome the difficulty, the author mapped the system output error to the nonlinear term of the framer, creating a framer of the extended Luenberger structure, and analyzed the coordination of the error system by the monotonic system theory. On this basis, a design method was proposed for the interval observer of the lure system. Then, the lure system and its observer systems were proved as asymptotically stable. Finally, it is proved that the observer system trajectory always followed the original state trajectory through the simulation under the different selections of set-valued mapping.

\section{Data Availability}

No data were used to support this study.

\section{Conflicts of Interest}

The authors declare that they have no conflicts of interest.

\section{Acknowledgments}

We are grateful to Prof Qian Fucai who made analysis for the paper. We also greatly appreciate Prof. Qian's useful discussion. This work was supported by the Foundation for Higher level Talents in Ankang University (2019AYQDZR01).

\section{References}

[1] T. Koide and T. Kodama, "Generalization of uncertainty relation for quantum and stochastic systems," Physics Letters A, vol. 382, no. 22, pp. 1472-1480, 2018.

[2] A. Gupta, C. Chakraborty, and B. Gupta, "Monitoring of epileptical patients using cloud-enabled health-IoT system," Traitement du Signal, vol. 36, no. 5, pp. 425-431, 2019.

[3] G. Jin, K. Bai, Y. Zhang, and H. He, "A smart water metering system based on image recognition and Narrowband Internet of Things," Revue d'Intelligence Artificielle, vol. 33, no. 4, pp. 293-298, 2019.

[4] B. Loutfi, Z. Samir, D. Ali, and G. M. Zinelaabidine, "Real time implementation of type-2 fuzzy backstepping sliding mode controller for twin rotor MIMO system (TRMS)," Traitement du Signal, vol. 36, no. 1, pp. 1-11, 2019.

[5] Y. Li, "Design and implementation of intelligent travel recommendation system based on internet of things," Ingénierie des systèmes d'information, vol. 23, no. 5, pp. 159-173, 2018.

[6] X. Li, C. Lin, and X. Xu, "A target tracking model for enterprise production monitoring system based on spatial information and appearance model," Traitement du Signal, vol. 36, no. 4, pp. 369-375, 2019.

[7] D. Efimov, L. Fridman, T. Raïssi, A. Zolghadri, and R. Seydou, "Interval estimation for LPV systems applying high order sliding mode techniques," Automatica, vol. 48, no. 9, pp. 2365-2371, 2012.

[8] Z. Zhang and S. Xu, "Observer design for uncertain nonlinear systems with unmodeled dynamics," Automatica, vol. 51, pp. 80-84, 2015.
[9] F. Zhu, "State estimation and unknown input reconstruction via both reduced-order and high-order sliding mode observers," Journal of Process Control, vol. 22, no. 1, pp. 296-302, 2012.

[10] J. Bodapati, V. Sajja, N. B. Mundukur, and N. Veeranjaneyulu, "Robust cluster-then-label (RCTL) approach for heart disease prediction," Ingénierie des systèmes d information, vol. 24, no. 3, pp. 255-260, 2019.

[11] I. R. Petersen, "Robust unobservability for uncertain linear systems with structured uncertainty," IEEE Transactions on Automatic Control, vol. 52, no. 8, pp. 1461-1469, 2007.

[12] K. Kumar and R. K. Mishra, "A robust mRMR based pedestrian detection approach using shape descriptor," Traitement du Signal, vol. 36, no. 1, pp. 79-85, 2019.

[13] P. Gahinet and P. Apkarian, "A linear matrix inequality approach toHo control," International Journal of Robust and Nonlinear Control, vol. 4, no. 4, pp. 421-448, 1994.

[14] W. P. M. H. Heemels, J. Daafouz, and G. Millerioux, "Observer-based control of discrete-time LPV systems with uncertain parameters \$ \$, IEEE Transactions on Automatic Control, vol. 55, no. 9, pp. 2130-2135, 2010.

[15] D. Ichalal and S. Mammar, "On unknown input observers for LPV systems," IEEE Transactions on Industrial Electronics, vol. 62, no. 9, pp. 5870-5880, 2015.

[16] D. Zhao, Q. Zhang, X. Li, and L. Kong, "Event-triggered $H \infty$ control for continuous-time nonlinear system," Advances in Neural Networks - ISNN 2015, vol. 9377, pp. 62-70, 2015.

[17] S. Yasini, M. B. Naghibi Sitani, and A. Kirampor, "Reinforcement learning and neural networks for multi-agent nonzero-sum games of nonlinear constrained-input systems," International Journal of Machine Learning and Cybernetics, vol. 7, no. 6, pp. 967-980, 2016.

[18] Y. Wang, V. Gupta, and P. J. Antsaklis, "On passivity of a class of discrete-time switched nonlinear systems," IEEE Transactions on Automatic Control, vol. 59, no. 3, pp. 692-702, 2014. 